\title{
Calculation Methodology of Power Plant Universal Cycle Using Low-grade Heat of Refrigerant
}

\author{
S.V. Zaika , D.A. Uglanov and S.S. Dostovalova \\ Samara University, Heat engineering and heat engines Department, Samara, Russia
}

\begin{abstract}
The problems of high energy loss exists in many industries and in many technological processes. The problem of not effective use of the energy potential of the refrigerants is the main problem, because the refrigerant loses its potential and the potential energy at the regasification upon receipt of the liquefied natural gas. Solution of this problem is using low-grade heat of the refrigerant. This investigation describes the creation of a design procedure of the installation of the cycle that uses low-grade heat of the refrigerant. The cycle that uses low-grade heat of the refrigerant has been described and calculation method has been developed.
\end{abstract}

\section{Introduction}

Currently, the use of low-temperature heat of cryogenic liquids in the industry is spreading rapidly. This is due to increasing volumes of liquefied natural gas, the general trend of energy saving and energy potential of cryogen in form of energy which has been expended on liquefaction [1]. In a typical regasification of cryogen using environmental heat or special sources heat, the working fluid acquires parameters of environment and loses lowtemperature potential. At the pressure equality, the working fluid wastes the potential energy of pressure gas [2]. Under real conditions, the conversion of thermal energy into other forms of energy takes place in the presence of the real environment with temperature $T_{e}$.

The environment can be used as a heat exchanger or a heat body. Figure 1 shows the Carnot cycle (A23BA and 1AB41). Cycles $\mathrm{T}_{1}^{+}>\mathrm{T}_{\mathrm{o.c}}=\mathrm{T}_{2}^{+}$are high-potential cycles. Cycles $\mathrm{T}_{1}^{-}>\mathrm{T}_{\mathrm{o} . \mathrm{c}}=\mathrm{T}_{2}^{-}$are low-potential cycles. Generally, the Carnot cycle has been used for an evaluation of thermal systems as a standard. Consider their effectiveness relative to the amount of heat input without regard to environmental effects [3-4].

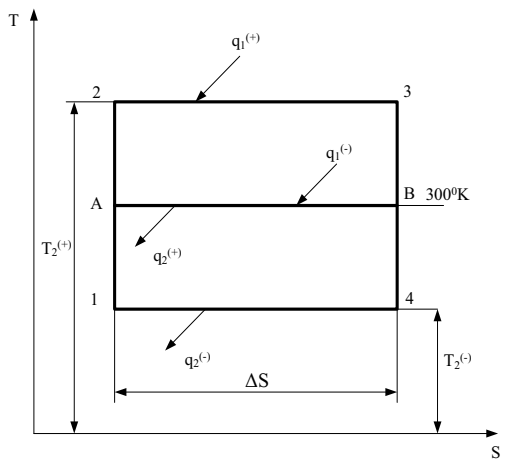

Figure 1. High-grade and low-grade Carnot cycles
The results indicate that Carnot cycle just serves as one form of the best cycles and only if the polytropic exponents (n) of the expansion and compression processes equal to each other, the efficiency of that cycle reaches the highest value, just that of Carnot cycle [5].

The purpose of this research is investigation of polytropic thermomechanical cycle working by lowgrade heat of cryogen. The formula of the efficiency of thermal energy converters contain coefficient $\eta_{0}=$ $\eta_{t_{n .0}} / \eta_{t_{\mathrm{K}}}$. For its evaluation we consider the theoretical cycle efficiency dependence of the most important cycle parameters [6].

For this purpose it is sufficient to investigate the generalized polytropic cycle (Figure 2), which is limited by the following assumptions: 1) processes are reversible; 2 ) the specific heat, and hence the polytropic indices are constant values; 3) working body - ideal gas; 4) heat supply is carried out during II, removal - during IV; 5) heat transfer in the process I is absent, although the process is considered as a polytropic (a consequence of the IPOA specificity, in which energy components are supplied either liquid, or in a precompressed state);6) Process III is considered to be adiabatic [7].

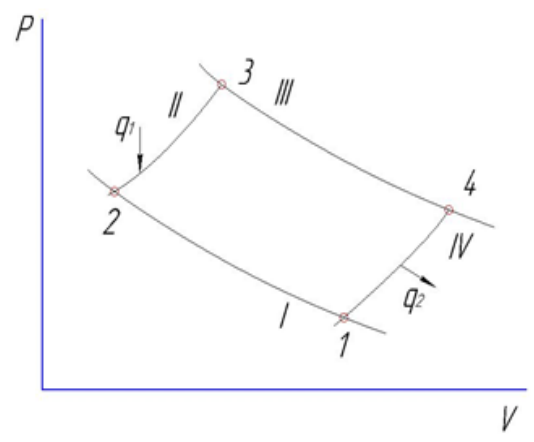

Figure 2. Polytropic cycle illustrated on a P-V diagram 
The equation $\eta_{t_{n .0}}$ of polytropic cycle should include the maximum-to-minimum cycle temperature ratio $T_{3} / T_{1}$ cycle and the pressure at point $4-\mathrm{p}_{4}$. For simplicity it can be taken $T_{1}=300 \mathrm{~K}$. When movement occurs in an environment with constant state parameters (on the surface of Earth, in space) backpressure $p_{\text {o.c }} \cong$ $p_{1} \leq p_{4}$, although ideally it should be equal $p_{\text {o.c }}=p_{4}[8]$. This case will be considered.

\section{Research of polytropic cycle}

If the working fluid parameters were set at point $1-\mathrm{p}_{1}, \mathrm{~V}_{1}$, $T_{1}$, polytropic index $n_{i}$, compression $\varepsilon_{\mathrm{ci}}$ or degrees of expansion $\varepsilon_{\mathrm{pi}}$, or pressure ratio $\pi\left(\pi=1 / \varepsilon_{p}^{n}=\varepsilon_{\mathrm{c}}^{n}\right.$, the efficiency will be equal to:

$$
\eta_{t_{p}}=1-\frac{c_{n_{I V}}\left(\pi_{I V}^{\frac{n_{I V}-1}{n_{I V}}}-1\right)}{c_{n_{I V} \varepsilon_{c_{I}}^{n-1}}\left(\pi_{I V}^{\frac{n_{I V}-1}{n_{I V}}}-1\right)}
$$

Let's introduce the ratio of minimum and maximum temperature cycle $T_{1} / T_{3}$, and make the conversions. Because the

Because the

$$
c_{n}=c_{V} \frac{n-k}{n-1}
$$

and

$$
\frac{T_{3}}{T_{1}}=\pi_{I}^{\frac{n_{I}-1}{n_{I}}} \cdot \pi_{I I}^{\frac{n_{I I}-1}{n_{I I}}}
$$

so

$$
\eta_{t_{n}}=1-\frac{T_{1}}{T_{3}} \frac{\frac{n_{I V}-k}{n_{I V}-1}\left(\pi_{I V}^{\frac{n_{I V}-1}{n_{I V}}}-1\right)}{\frac{n_{I I}-k}{n_{I I}-1}\left(\pi_{I I}^{\frac{n_{I I}-1}{n_{I I}}}-1\right) \cdot \pi_{I I}^{\frac{n_{I I}-1}{n_{I I}}}}
$$

or

$$
\eta_{t_{n}}=1-\frac{T_{1}}{T_{3}} \frac{\frac{n_{I V}-k}{n_{I V}-1}\left(\varepsilon_{c_{I V}}^{n_{I V}-1}-1\right)}{\frac{n_{I I}-k}{n_{I I}-1}\left(1-\varepsilon_{p_{I I}}^{n_{I I}-1}\right)}
$$

Formula (1) is convenient to use when $\varepsilon_{p_{I I}}=\varepsilon_{p_{V I}}=$ 1 and $n_{I I}=n_{I V}=\infty$, at isochoric process II-III and IV-I, the expression (2) - when $\pi_{I I}=\pi_{I V}=1$ and $n_{I I}=n_{I V}=$ 0 . All intermediate cases, they are equal and the choice should be made depending on the researcher's interests to influence $\pi$ or $\varepsilon_{\mathrm{c}}=1 / \varepsilon_{p}$. Substituting in (1) and (2) the values of the polytropic indexes and characteristics corresponding to the Carnot cycle, converts them to the Carnot efficiency $\eta_{t_{\Pi}}=\eta_{t_{k}}=1-\frac{T_{1}}{T_{3}}$.

Let's investigate the expression (1). The cases has practical interest when $\pi_{I I}>1,0<n_{I I}<1 ; 1<n_{I I} \leq$ $\infty$ and $\pi_{I V}>1,0<n_{I V}<1 ; 1<n_{I V} \leq \infty$.
From the above $n_{I V}=f\left(\pi_{I}, \pi_{I I}, \pi_{I V}, n_{I}, n_{I I}, k\right)$. This relation could be easily represented in the form

$$
n_{I V}=\frac{\lg \frac{p_{4}}{p_{1}}}{\lg \frac{\vartheta_{1}}{\vartheta_{4}}}=\frac{\lg \pi_{I V}}{\lg \pi_{I}^{\left(\frac{1}{n_{I}}-\frac{1}{k}\right)} \cdot \pi_{I I}^{\left(\frac{1}{n_{I I}}-\frac{1}{k}\right)} \cdot \pi^{\frac{1}{k}}}
$$

It allows to identify and, most importantly, establish relationship with $\pi_{I V}$ for each case. Communication between $n_{I V}$ and $T_{1} / T_{3}$ can be obtained in the following form:

$$
n_{I V}=\frac{\lg \pi_{I V}}{\lg \frac{T_{1}}{T_{3}}\left(\pi_{I} \pi_{I I}\right)^{1-\frac{1}{k}} \pi_{I V}^{\frac{1}{k}}}
$$

For analysis of (1) values $n_{I V}$ and $\pi_{I V}$ can be specified, taking into account, that the ratios of (3) and (4) are not broken (since other parameters and characteristics can change accordingly). However, it's more interesting to obtaine the results, taking into account the impact of the dependencies (3), (4).

Considering, that under the real conditions $\left(\pi_{I}, \pi_{I I}\right) \gg$ $\pi_{I V}$, it is permissible to restrict the study in cases, where $\left(\pi_{I}, \pi_{I I}\right)>\pi_{I V}$. The maximum take equal $\pi_{I V}=1000$ (the case is unreal). We introduce into the formula (I) ratio:

$$
C_{c}=\frac{1-\frac{1}{n_{I I}}}{1-\frac{1}{k}} \cdot \frac{1-\frac{1}{n_{I I}}}{1-\pi_{I I}^{\left(\frac{1}{n_{I I}}-1\right)}}=\frac{1-\frac{1}{n_{I I}}}{1-\frac{1}{k}} \cdot \frac{1}{1-\varepsilon_{p_{I I}}^{1-n_{I I}}}
$$

After that, the equation takes the form:

$$
\eta_{t_{n}}=1-\frac{T_{1}}{T_{3}}\left[\frac{1-\frac{k}{n_{I V}}}{1-\frac{1}{n_{I V}}}\left(\pi_{I V}^{\frac{n_{I V}-1}{n_{I V}}}-1\right) C_{c}\right]
$$

Investigating $n_{I V}$ change and choose $C_{c}$ near $n_{I I}=\infty$ and $n_{I I}=0$ due to the fact that these values are typical for gas and for vapor-liquid cycles. Change $n_{I V}$ sign occurs in the transition of its value through $n_{I V}= \pm \infty$, is suitable, as is known, isochoric process. These values $n_{I V}$ shifted depending on the temperature (and pressure $\pi$ ), which is easy to determine if the condition that $\lg \frac{T_{1}}{T_{3}}\left(\pi_{I} \pi_{I I}\right)^{1-\frac{1}{k}} \pi_{I V}^{\frac{1}{k}}=0$.

So we can tacen this eqn:

$$
T_{3}=T_{1}\left(\pi_{I} \pi_{I I}\right)^{1-\frac{1}{k}} \pi_{I V}^{\frac{1}{k}}
$$

From the equation it follows that

$$
T_{1}=\frac{T_{3}}{\left(\pi_{I} \pi_{I I}\right)^{1-\frac{1}{k}} \pi_{I V}^{\frac{1}{k}}}=\frac{T_{3}\left(\pi_{I} \pi_{I I}\right)}{\pi_{I V}^{\frac{1}{k}}}
$$


Using the above relations, we can investigate $\eta_{t_{\text {п.о }}}$ according to the equation (5). It's enough to take $n_{I V}$ in the range corresponding to $\pi_{I V}$ and $T_{3}$. Values are selected according to $C_{c}$ that can be defined for known $\pi_{I} \pi_{I I}$ and $T_{1} / T_{3}\left(\frac{T_{3}}{T_{1}}=\pi_{I}^{\frac{n_{I}-1}{n_{I}}} \cdot \pi_{I I}^{\frac{n_{I I}-1}{n_{I I}}}\right)$.

$$
\pi_{I I}=\frac{1}{\varepsilon_{p_{I I}}^{n_{I I}}}=\left[\frac{\pi_{I} \pi_{I I}}{\left(\frac{T_{1}}{T_{3}}\right)^{\frac{1}{n_{I I}}-1}}\right] \frac{\frac{1}{n_{I I}}-1}{\left(\frac{1}{n_{I I}}-1\right)+\left(\frac{1}{n_{I}}-1\right)}
$$

$C_{c} \rightarrow \infty$ at $n_{I I} \rightarrow k$ and $C_{c} \rightarrow 0$ for $n_{I I} \rightarrow \infty$ and $n_{I I} \rightarrow 0$. Increasing $\pi_{I I} \gg 3 \div 10$, especially at high $n_{I I} \gg k$, doesn't have significant impact on $C_{c}$. Conversely, at low $\pi_{I I} \ll 3 \div 10$ absolute value $C_{c}$ sharply increases. Since the process II-III can be adiabatic (as the heat is being supplied by condition) and can not be $n_{I I}=k$. Therefore, it makes no sense to consider the case when $C_{c}=\infty$. Under the real conditions $n_{I I} \neq \infty$ and $n_{I I} \neq 0$ is sufficient to investigate the change $\eta_{t_{\Pi}}$ at $C_{c}=-50 \div+50$. If we consider that usually $n_{I I}>k$, then the actual values can be limited by the quantities $-5 \div+5$.

In order to determine the range of variation $C_{c}$, we should determine the values $C_{c k}$, in which $\eta_{t_{n}}=\eta_{t_{\mathrm{K}}}$, т.e.

$$
C_{c k}=\left[\frac{1-\frac{k}{n_{I V}}}{1-\frac{1}{n_{I V}}}\left(\pi_{I V}^{1-\frac{1}{n_{I V}}}-1\right)\right]^{-1}
$$

\section{Change polytropic cycle efficiency depending on conditions}

Using these relations, curves were built (Figure $3-6$ ), which express the relationship (the V), as an example for $\mathrm{k}=1,6$. If necessary, these charts can be constructed for any value of argument $\eta_{t_{n .0}}$. Two cases are considered: $n_{I I}=0,5$ and $n_{I V}=0,55$ (Figure $3-4$ ); $n_{I I}=1,2$ and $n_{I V}=0,85$ (Figure 5-6).

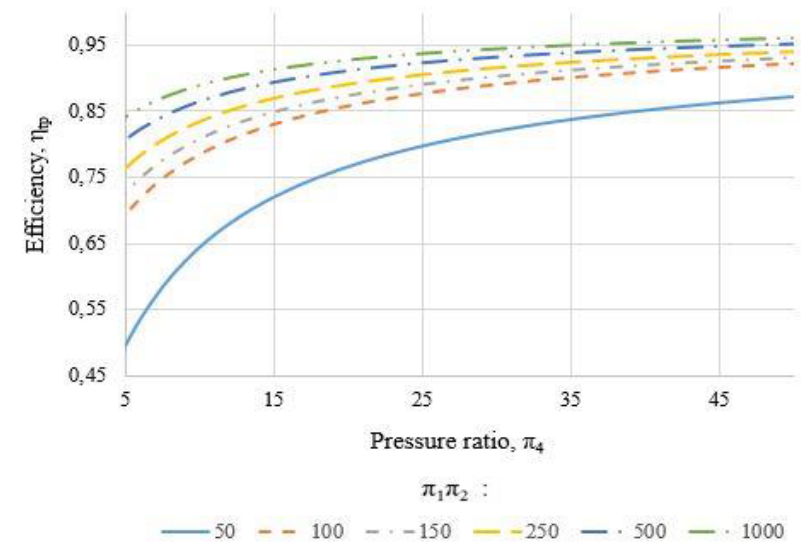

Figure 3. The dependence of the efficiency of the pressure ratio at $n_{I I}=0,5$ and $n_{I V}=0,55$

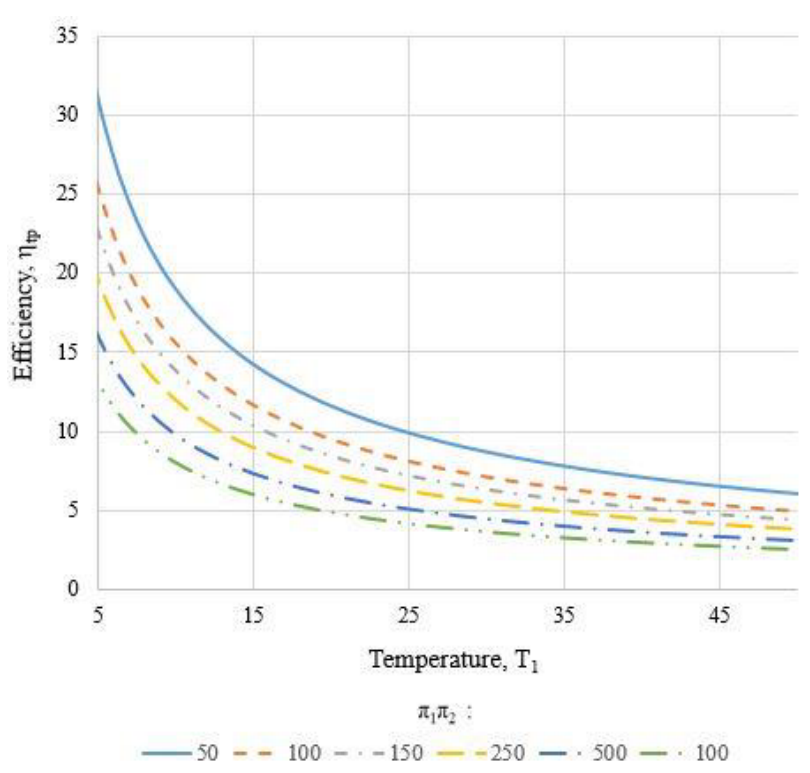

Figure 4. The dependence of the efficiency of the temperature at $n_{I I}=0,5$ and $n_{I V}=0,55$

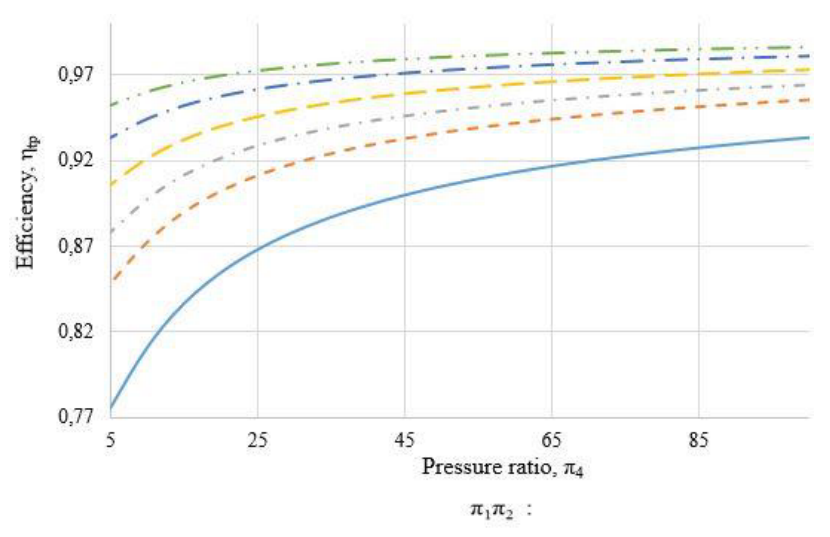

$-50--\cdot 100-\cdot-150--250-\cdot 500-\cdot 1000$

Figure 5. The dependence of the efficiency of the pressure ratio at $n_{I I}=1,2$ and $n_{I V}=0,85$

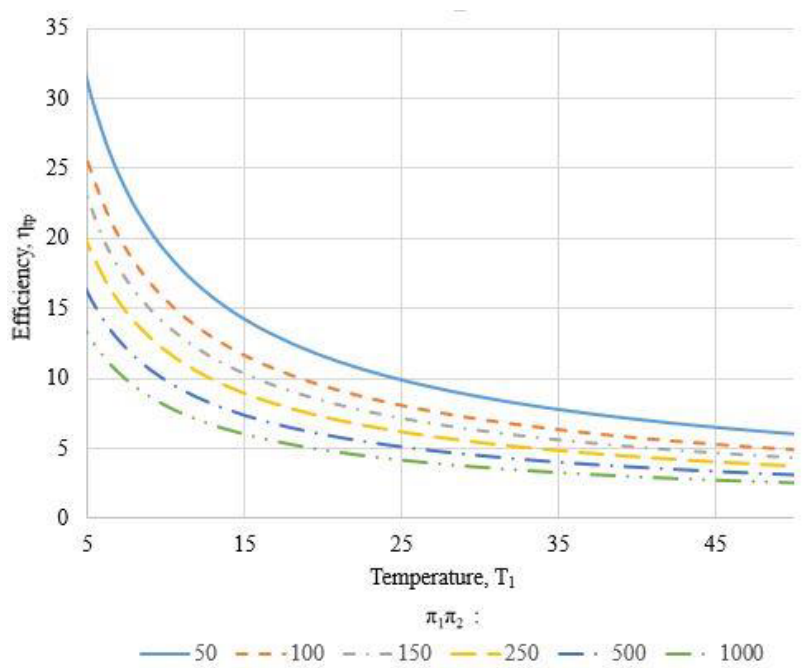

Figure 6. The dependence of the efficiency of the temperature at $n_{I I}=1,2$ and $n_{I V}=0,85$

The above graph shows: 1) complex and different dependence of the efficiency $\eta_{t_{\Pi}}$ from $\pi_{I V}$, allowing in each case to find the optimal values $k, \pi_{I}, \pi_{I I}$ and $T_{1} / T_{3}$ 
for $\pi_{I V}$ data; 2) significant differences of this dependence from the dependence $\eta_{t_{k}}=f\left(T_{1}, T_{2}, \Delta T\right)$. They show that the increase of economic efficiency is not enough to strive to increase the values of the polytropic exponent, since under certain conditions it can lead to the opposite result.

Analysis graphs below allows to choose the most advantageous values $\eta_{t_{\Pi}}$ and $\eta_{t_{k}}$ for each particular case, and hence the value of the coefficient of completeness of the reversible conversion of energy $\eta_{\mathrm{o}}$, as well as match $P T(k, R)$. The relative efficiency of actual cycles depending on the working bodies compared to the Carnot cycle efficiency can be estimated by the number of work.

\section{Conclusions}

A method for calculating the universal thermodynamic cycle power plant using low-potential energy of cryogen was developed.

Main characteristics of dependence and, as well as their changes depending on the conditions were found. The following main conclusions can be distinguished on the basis of the study:

1. The calculations for two cases: $n_{I I}=0,5$ and $n_{I V}=0,55 ; n_{I I}=1,2$ and $n_{I V}=0,85$ were made. The results confirm the higher $\pi_{I V}$, the higher $\eta_{t_{n}}$. It can be concluded that increase $T_{1}$ leads to decrease $\pi_{I V}$, and hence to decrease $\eta_{t_{n}}$.

2. The higher $n_{I I}$ and $n_{I V}$, the higher the efficiency of the universal cycle power plant that uses low-grade heat of cryogenic.

Studies have been conducted polytropic cycle. Change polytropic cycle efficiency depending on conditions was analysed and described.

This research gives a new insight into cycles using low-grade heat of refrigerant, which is of great significance in to solve thermodynamic optimization problems. So this topic is really worth deep future investigations.

\section{References}

1. Hung T. Ahlborn B. Waste heat recovery of organic Rankine cycle using dry fluids// Energy Convers. Manage., 42 (2001), pp. 539-553

2. Walraven D., Laenen B., D'haeseleer W. Comparison of thermodynamic cycles for power production from low-temperature geothermal heat sources // Energy Convers. Manage., 66 (2013), pp. 220-233

3. Hansaem Park, Min Soo Kim. Performance analysis of sequential Carnot cycles withfinite heatsources and heat sinks and its application in organic Rankine cycles // Energy (V.99). 2016, pp. 1 - 9

4. Dovgyallo A.I., Nekrasova S.O., Sarmin D.V., Uglanov D.A. Imitations tests of throttle cooling system with cryogenic refueling tank and its comparative characteristics // Applied Physics. 2013. Issue 4, 2013, Pages. P. 54-59

5. Rui Shang, Yin Zhang, Wenxing Shi, Xin Wang, Yinping Zhang. Fresh Look and Understanding on Carnot Cycle // Energy Procedia. 2014. Pages 28982901

6. Biryuk V.V., Gorshkalev A.A., Kayukov S.S., Uglanov D.A. Gas-Dynamic Analysis of Processes in a Small-Sizes Two-Stroke Combustion Engine // The Open Mechanical Engineering Journal. 2014. 8. P. 441-444

7. Biryuk V.V., Tsapkova A. B., Shimanov A.A. Numerical modeling of cyclone machine for cleaning gas generator gases, Key Engineering Materials. 2016. Volume 685. P. 153-157

8. Nekrasova, S.O., Uglanov, D.A.,Shimanov, A.A. Comparative analysis of calculation models of pulse tube thermoacoustic refrigerator, 4th International Conference for Young Scientists High Technology: Research and Applications, HTRA 2015, 21 - 24 April 2015, Publisher: Trans Tech Publications Ltd, 2016 г., P. 3-7 